\title{
Configuración anatómica del sistema canalicular de la raíz mesiovestibular del primer molar maxilar
}

\section{Anatomical configuration of canalicular system in mesiobuccal root of maxillary first molar}

\author{
Betancourt P*, Aracena Rojas S**, Navarro Cáceres P***, Fuentes $\mathrm{R}^{* * * *}$
}

\section{RESUMEN}

Introducción: El primer molar maxilar permanente es el diente que presenta mayor complejidad y variación morfológica, que se refleja en la más alta tasa de fracaso clínico. Las principales causas de su fracaso en el tratamiento endodóntico es la imposibilidad de localizar el canal mesiovestibular secundario y el desconocimiento de su morfología. El objetivo de este estudio fue determinar, in vitro, la configuración del canal MB2 presente en la raíz mesiovestibular del primer molar maxilar, mediante radiografías periapicales.

Material y métodos: Se seleccionaron 62 primeros molares maxilares. Se procedió a tomar radiografías iniciales periapicales de cada diente en sentido mesiodistal y vestíbulo-palatino. Los molares fueron trepanados y los canales fueron permeabilizados. Para evidenciar la anatomía de los canales radiculares, se posicionó en el canal MB1 una lima Headstrom $\mathrm{n}^{\circ}$ 0,8 o 10 y en el canal MB2 una lima k-flex $\mathrm{n}^{\circ}$ 0,8 o 10. Los especímenes fueron sometidos a una radiografía periapical, en sentido mesiodistal y vestíbulo-palatino.

Resultados: El 30,38\% de los dientes presentó un sistema de canales tipo I, 41,07\% correspondió al tipo II, un $28,57 \%$ al tipo III y un $0 \%$ al tipo IV. La frecuencia del canal mesiovestibular secundario fue de un 69,64\%.

Discusión: El clínico debe tener la convicción de realizar la exploración en búsqueda del canal MB2, logrando un adecuado acceso endodóntico que permita una visualización correcta, utilizar herramientas como el microscopio, ultrasonido o TCHC para su detección y estudio morfológico.

Palabras clave: Primer molar maxilar permanente, canal secundario, raíz mesiobucal.

\section{SUMMARY}

Introduction: Permanent maxillary first molar is the tooth that presents the most complex morphological variation, which is reflected in a higher rate of clinical failure. One of the main causes of endodontic treatment failure is the inability to locate the secondary mesiobuccal canal and the ignorance of their morphology. The aim of this study was to determine, in vitro, the configuration of MB2 canal present in the mesiobuccal root of the first maxillary molar, through periapical radiographs.

Methods: Sixty-two maxillary first molars were selected. After this, we proceeded to take initial periapical radiographs of each tooth in mesio-distal and bucco-palatal faces. The molars were approached until pulp chamber and canals were permeabilized. In order to demonstrate the anatomy of root canals, a Hedstrom file $\mathrm{n}^{\circ} 0,8$ or 10 was positioned over the MB1 canal and a K- flex file $n^{\circ} 0,8$ or 10 over the MB2 canal. Specimens were subjected to a periapical radiograph as a final control in the same mesio-distal and bucco-palatal faces.

* Magíster en Odontología. Centro de Investigación CIMOFIR, Facultad de Odontología. Universidad de La Frontera, Temuco, Chile.

** Especialista en Endodoncia. Universidad Mayor, Temuco, Chile.

*** Bioestadístico. Facultad de Odontología, Universidad de La Frontera, Temuco, Chile.

**** Doctor en Odontología. Profesor Asociado. Centro de Investigación CIMOFIR, Facultad de Odontología. Universidad de La Frontera, Temuco, Chile. 
Results: $30,38 \%$ of teeth had type I canal system, $41,07 \%$ were type II, $28,57 \%$ had type III and $0 \%$ type IV. The frecuency of the second mesiobuccal canal was $69,64 \%$.

Discussion: The clinician must be satisfied to perform exploration in search of $M B 2$ canal, making an adequate endodontic access to allow a correct display, to use tools like microscope, ultrasound or TCHC for its detection and morphological study.

Key words: First permanent maxillary molar, second canal, mesiobuccal root.

Fecha de recepción: 22 de abril de 2014.

Aceptado para publicación: 25 de mayo de 2014.

Betancourt P, Aracena Rojas S, Navarro Cáceres P, Fuentes R. Configuración anatómica del sistema canalicular de la raíz mesiovestibular del primer molar maxilar. Av. Odontoestomatol 2015; 31 (1): 11-18.

\section{INTRODUCCIÓN}

El primer molar maxilar permanente (1MM) es el diente que presenta mayor complejidad y variación morfológica (1), que se refleja en la más alta tasa de fracaso clínico (2), lo que hace de él un desafío constante. Se describe anatómicamente con tres raíces, mesiovestibular (MB), distovestibular (DB), y palatina $(P)$; aunque se han reportado variaciones, oscilando entre 1 a 5 raíces distintas y hasta 7 canales en una raíz (3). Diversos estudios $(4,5)$, han demostrado que el canal mesiovestibular secundario (MB2) está presente en más del $50 \%$ de $1 \mathrm{MM}$, por lo tanto, se deben utilizar todos los métodos posibles para localizar y detectar los canales del sistema radicular, así como también estudiar su morfología.

Para clasificar los sistemas de canales radiculares en la raíz mesiovestibular del 1MM, Weine (6) describe cuatro tipos de configuraciones radiográficas, Tipo I: desde la cámara pulpar sale un único canal hasta la salida apical, Tipo II: desde la cámara pulpar salen dos canales separados, pero se fusionan en la zona apical para formar un solo canal. Tipo III: desde la cámara pulpar emergen 2 canales separados y se mantienen así hasta su salida en apical. Tipo IV: canal único en la salida de la cámara y termina en dos canales separados en apical.

Diferentes métodos han sido adoptados para estudiar la anatomía canalicular de la raíz mesiovestibular del $1 \mathrm{MM}$, in vivo, tales como TCHC (7), microscopio endodóntico (3), magnificación y radiografías periapicales (8). Así también, técnicas in vitro, tales como tinción (9), cortes de secciones transversales radiculares (10), examen radiográfico (6), lupa de aumento (10), microscopio quirúrgico clínico (11), ultrasonido (12), microscopio electrónico de barrido (10), TCHC $(4,5)$ y micro tomografía computarizada (13), reportándose diferencias en los resultados de localización de éstos, según la técnica y condiciones utilizadas, considerando una tasa promedio de detección del canal MB2 in vivo de un 40\%, en comparación con el $80 \%$ descrito en estudios in vitro (2). Gracias al avance de la tecnología, en particular, puntas ultrasónicas, magnificación y exámenes imagenológicos, se ha producido un aumento de localización del canal MB2 en los últimos años. Sin embargo, el canal MB2, con frecuencia no es detectado por el clínico en la práctica diaria, y aún cuando se localiza, se desconoce su morfología (14). El objetivo de este estudio fue determinar, in vitro, la configuración del canal MB2 presente en la raíz mesiovestibular del $1 \mathrm{MM}$, utilizando la clasificación de Weine (6), mediante radiografías periapicales.

\section{MATERIAL Y MÉTODO}

El diseño utilizado en este estudio fue descriptivo observacional de corte transversal. Se seleccionaron $621 M M$, de pacientes de ambos sexos, extraídos por indicación de caries, periodontal u ortodóntica en el Policlínico de cirugía, Universidad de La Frontera, Temuco, Chile, entre los meses de enero y agosto del año 2013. Los especímenes fueron almacenados en envases individuales, numerados y etiquetados con sexo y edad del paciente, que contenían 
Clorhexidina al 0,12\% ( Perioaid T®, Dentaid, Cerdanyola, España). Se utilizaron los dientes que cumplieron los siguientes criterios de inclusión: edad entre 10 y 75 años y formación radicular completa. Se excluyeron los molares con canales radiculares con relleno endodóntico, calcificados, rehabilitados mediante prótesis fija, evidencia de radectomía o cirugía periapical. Las muestras fueron sometidas a una limpieza en la parte externa de la raíz, por medio de puntas de ultrasonido Endo Pro Ultra de circonio Satelec ${ }^{\circledR}$ (Dentsply Maillefer ${ }^{\circledR}$, Ballaigues, Suiza ) y de una cureta 7/8, Gracey (Hu-Friedy ${ }^{\circledR}$, Chicago, EE.UU), para eliminar restos de ligamento periodontal y cálculos de la superficie radicular.

Para estandarizar la posición de los dientes en las distintas tomas radiográficas del estudio, se acondicionó la superficie externa radicular en las regiones distal y palatina, y se aplicó composite Filtek A3 Z 250 (3m®, St Paul, USA).

Posteriormente, se procedió a tomar radiografías iniciales periapicales (Kodak Ultra Speed $\AA$, DF 58, New York, EEUU) de cada diente en sentido mesiodistal y vestíbulo-palatino, mediante equipo de rayos x (Procion ion 70 x, serie 11211232017-B, São Paulo, Brasil). Se utilizó la técnica del paralelismo, a una distancia estandarizada de 47,2 cm dada por una mesa de posicionamiento radiográfico (Fig. 1), donde la posición del tubo y de la película radiográfica fue la misma para todas las muestras (15). La potencia utilizada correspondió a $70 \mathrm{kV}$ y la exposición a 0,63 segundos en modo adulto. Las placas radiográficas fueron reveladas en un revelador automático (Perio Mat Plus ${ }^{\circledR}$, New York, USA). Una vez realizado este procedimiento, los molares fueron trepanados por medio de una fresa redonda de diamante $n^{\circ} 2$ de alta velocidad, fresa redonda de carbide $n^{\circ} 2$ de baja velocidad y una fresa troncocónica de alta velocidad con punta inactiva (Endozeta, Dentsply Maillefer ${ }^{\circledR}$, Ballaigues, Suiza).

Se procedió a localizar los canales a nivel cameral, realizando un acceso inicial de forma romboidal, lo que permitió un acceso directo al canal MB2, en las situaciones en que se encontraba presente. Mediante un quelante (Glyde File prep, Dentsply Maillefer ${ }^{\circledR}$, Ballaigues, Suiza), se permeabilizaron el o los canales radiculares de la raíz mesiovestibular, mediante

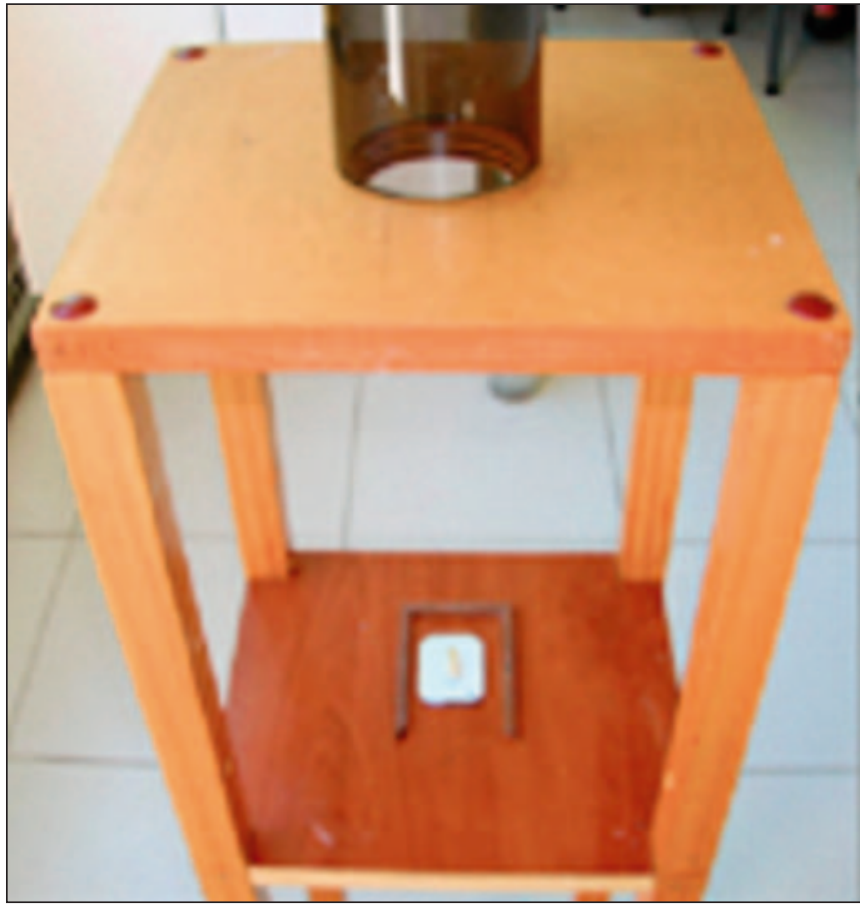

Fig. 1. Mesa de toma radiográfica estandarizada, con una distancia foco objeto de $47,2 \mathrm{~cm}$, donde la posición del tubo y de la película radiográfica fue la misma para todas las muestras.

una lima $\mathrm{k}$ 0,6, 0,8 o 0,10, de acuerdo con la morfología canalicular, hasta que fueron claramente visibles, sobrepasando la constricción apical. Luego se restó un milímetro de la longitud medida, obteniendo la longitud de trabajo.

Para evidenciar la disposición anatómica de los canales radiculares, se posicionó en el canal MB1 una lima Headstrom $n^{\circ}$ 0,8 o 10 y en el canal MB2 una lima k-flex $n^{\circ}$ 0,8 o 10 (Dentsply Maillefer ${ }^{\circledR}$, Ballaigues, Suiza) y se tomó una radiografía periapical, en sentido mesiodistal y vestíbulo-palatino, utilizando el mismo protocolo que para la radiografía inicial. Así, todas las radiografías fueron analizadas por dos endodoncistas previamente calibrados y los datos registrados (sexo, edad, lado en la hemiarcada del diente, presencia o ausencia canal MB2, tipo configuración canal MB2) fueron ingresados en una tabla Excel doble entrada.

El Análisis Estadístico se realizó utilizando un programa de software SPSS/PC + versión 20.0, SPSS, Chicago, IL. Se determinó la estadística descriptiva y se calcularon las medias \pm SD. Fueron utilizadas la 
prueba chi cuadrado de Pearson, el test exacto de Fisher y la prueba $t$ de Student para muestras independientes. Un valor de $\mathrm{p}<0,05$ fue elegido como umbral de significación estadística.

\section{RESULTADOS}

De los 62 dientes estudiados, 6 de ellos fueron descartados debido a calcificaciones, obteniendo una muestra total de 56 dientes. Se observó una frecuencia del canal MB2 en 39 dientes $(69,64 \%)$.

Se utilizó la clasificación de Weine (6), quién para categorizar las variables morfológicas en la raíz mesiovestibular del $1 \mathrm{MM}$, posicionó limas endodónticas en los canales de la raíz mesiovestibular y visualizó la configuración canalicular a través de radiografías periapicales.

Se obtuvo los siguientes resultados (Tabla 1): 17 dientes $(30,38 \%)$ presentaron un sistema de canales tipo I, un único canal hasta la salida apical y donde no se observó canal MB2 (Fig. 2), 23 (41,07\%) correspondieron al tipo II, desde la cámara pulpar salen dos canales separados, pero se fusionan en la zona apical para formar un solo canal (Fig. 3) y $16(28,57 \%)$ mostraron un sistema tipo III, desde la cámara pulpar emergen 2 canales separados y se mantienen así hasta su salida en apical (Fig. 4). Ninguno de los

\begin{tabular}{|c|c|c|}
\hline \multicolumn{3}{|c|}{$\begin{array}{c}\text { TABLA 1.- PORCENTAJE DE } \\
\text { CONFIGURACIONES CANALICULARES } \\
\text { PRESENTES EN LA RAÍZ } \\
\text { MESIOVESTIBULAR DEL PRIMER MOLAR } \\
\text { MAXILAR, UTILIZANDO LA CLASIFICACIÓN } \\
\text { DE WEINE, MEDIANTE RADIOGRAFÍAS } \\
\text { PERIAPICALES EN MUESTRA, POBLACIÓN } \\
\text { ESPECÍFICA DE TEMUCO, CHILE }\end{array}$} \\
\hline Clasificación & Número de la muestra & $\%$ \\
\hline $\begin{array}{l}\text { Tipo I (un canal) } \\
\text { Tipo II } \\
\text { Tipo III } \\
\text { Tipo IV }\end{array}$ & $\begin{array}{r}17 \\
23 \\
16 \\
0\end{array}$ & $\begin{array}{r}30,38 \\
41,07 \\
28,57 \\
0,00\end{array}$ \\
\hline Total & 56 & 100,00 \\
\hline
\end{tabular}

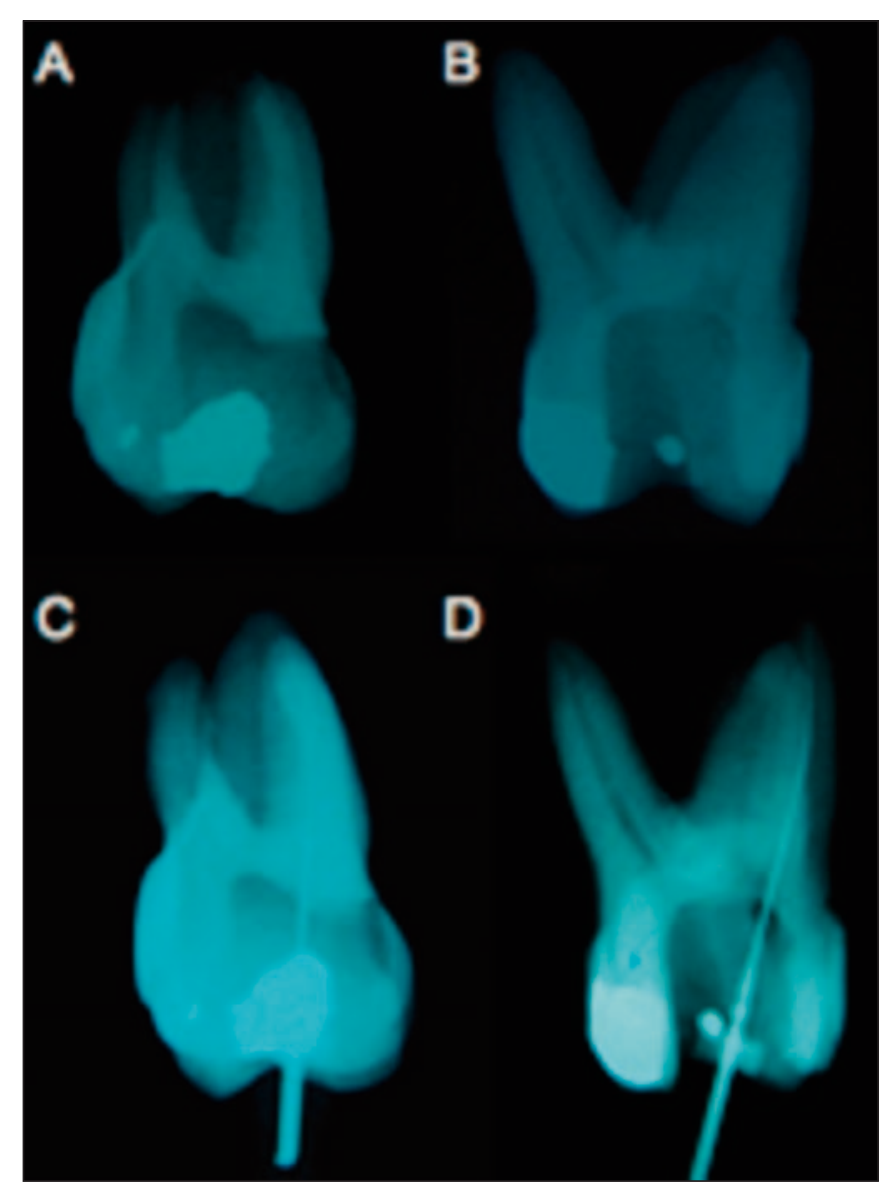

Fig. 2. Sistema de canales tipo I de Weine. Desde la cámara pulpar sale un único canal hasta la salida apical. A) Radiografía preoperatoria vestíbulo-palatino. B) Radiografía preoperatoria mesiodistal. C) Lima posicionada en canal MB1 vista desde ángulo vestíbulo-palatino. D) Lima posicionada en canal MB1 visualizada desde ángulo mesiodistal. Presencia de canal único en la raíz mesiovestibular del primer molar maxilar.

molares analizados presentó un sistema de canales tipo IV.

La distribución porcentual del canal MB2 según sexo fue mayor en mujeres $(51,3 \%)$ que en hombres $(48,7 \%)$. En cuanto a la lateralidad, el lado derecho mostró un porcentaje levemente mayor $(51,3 \%)$ que el izquierdo (48,7\%).

El promedio de edad en que se encontró en canal MB2 fue de 34 años.

Al analizar los datos que asocian la presencia del MB2 con la edad a través de la prueba $t$ de Student para muestras independientes, se obtuvo un valor 


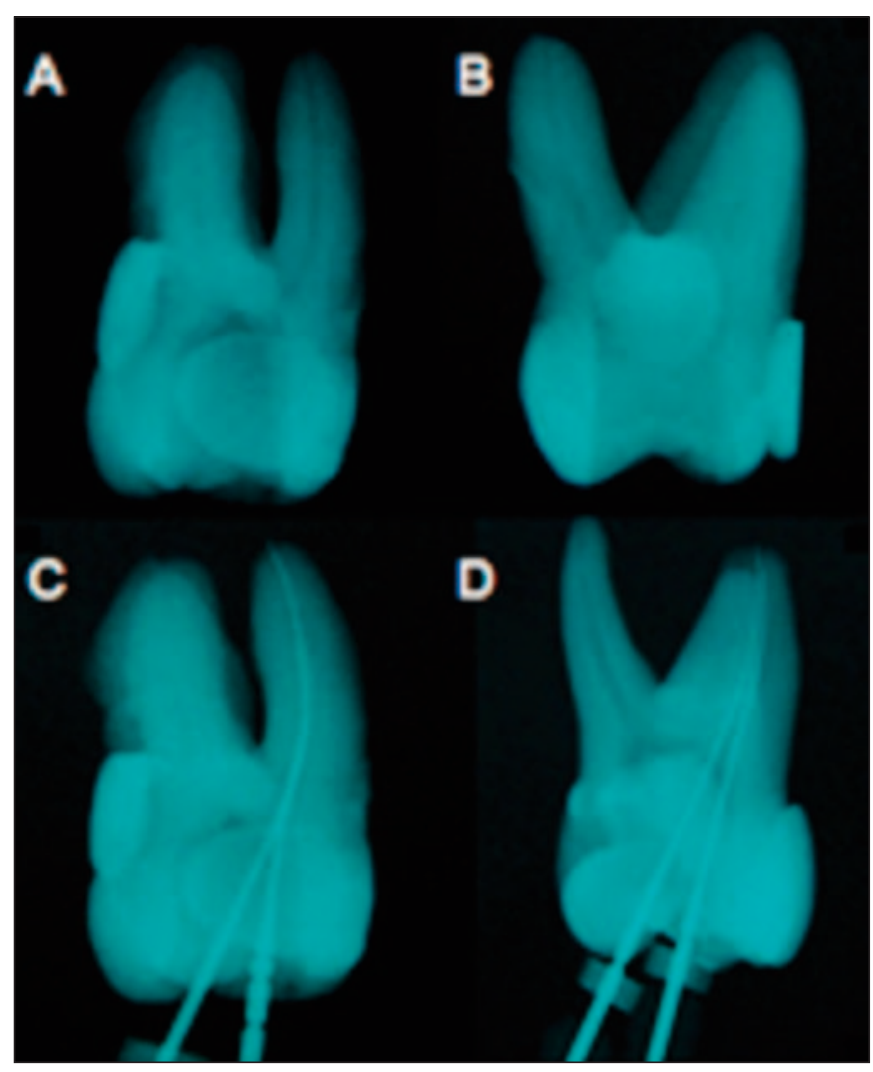

Fig. 3. Sistema de canales tipo II de Weine. Desde la cámara pulpar salen dos canales separados, pero se fusionan en la zona apical para formar un solo canal. A) Radiografía preoperatoria vestíbulopalatino. B) Radiografía preoperatoria mesiodistal. C) Limas posicionadas en canal MB1 y MB2 visualizadas desde ángulo vestíbulo-palatino. D) Limas posicionadas en canal MB1 y MB2 visualizadas desde ángulo mesiodistal. Las limas indican dos canales separados, pero que se fusionan en la zona apical para formar un solo canal en la raíz mesiovestibular del primer molar maxilar.

$p=0,006$, lo cual indica que existen diferencias estadísticamente significativas (Tabla 2). Según la prueba chi cuadrado de Pearson, no se observaron diferencias estadísticamente significativas al analizar la relación entre presencia del MB2 según sexo $(p=$ $0,771)$ y lateralidad $(p=0,353)$ (Tablas 3 y 4$)$.

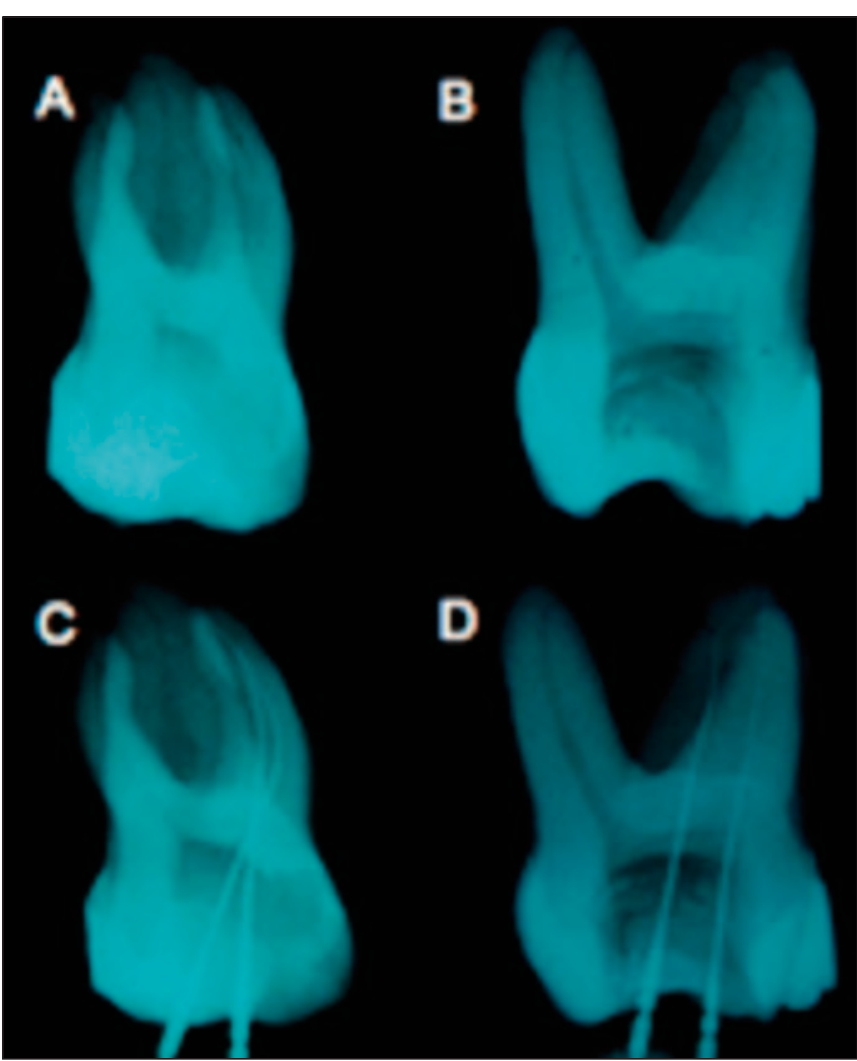

Fig. 4. Sistema de canales tipo III de Weine. Desde la cámara pulpar emergen 2 canales separados y se mantienen así hasta su salida en apical. A) Radiografía preoperatoria vestíbulo-palatino. B) Radiografía preoperatoria mesiodistal. C) Limas posicionadas en canal MB1 y

MB2 visualizadas desde ángulo vestíbulo-palatino. D) Limas posicionadas en canal MB1 y MB2 visualizadas desde ángulo mesiodistal. Las limas indican dos canales separados que se mantienen así hasta su salida en apical en la raíz mesiovestibular del primer molar maxilar.

\section{DISCUSIÓN}

El 1MM muestra variaciones en el sistema de canales radiculares de la raíz mesiovestibular, sin embargo, aunque es uno de los dientes más estudiados, es el que refleja la más alta tasa de fracaso clínico (2),

\section{TABLA 2.- PROMEDIO DE EDAD ASOCIADO A LA PRESENCIA DEL CANAL MB2 EN MUESTRA, POBLACIÓN ESPECÍFICA DE TEMUCO, CHILE}

\begin{tabular}{|ll|c|c|c|c|c|}
\hline MB2 & & $\mathbf{n}$ & Media & Desviación típ. & Error típ. de la media & $\mathbf{p}$ \\
\hline \multirow{2}{*}{ Edad } & Ausencia & 17 & 44,1765 & 10,03 & 2,4348 & $\mathbf{0 , 0 0 6}$ \\
& Presencia & 39 & 33,5385 & 13,63 & 2,1829 & \\
\hline
\end{tabular}




\begin{tabular}{|c|c|c|c|c|}
\hline \multirow{2}{*}{ Sexo } & \multicolumn{2}{|c|}{ MB2 } & \multirow{2}{*}{ Total } & \multirow{2}{*}{$\mathrm{p}$} \\
\hline & Ausencia & Presencia & & \\
\hline $\begin{array}{l}\text { Femenino } \\
\text { Masculino }\end{array}$ & $\begin{array}{ll}8 & (47,1 \%) \\
9 & (52,9 \%) \\
\end{array}$ & $\begin{array}{ll}20 & (51,3 \%) \\
19 & (48,7 \%)\end{array}$ & $\begin{array}{ll}28 & (50,0 \%) \\
16 & (50,0 \%)\end{array}$ & 0,631 \\
\hline Total & $17(100,0 \%)$ & $39(100,0 \%)$ & $56(100,0 \%)$ & \\
\hline
\end{tabular}

\begin{tabular}{|c|c|c|c|c|}
\hline \multicolumn{5}{|c|}{$\begin{array}{l}\text { TABLA 4.- DISTRIBUCIÓN PORCENTUAL DE FRECUENCIA DEL CANAL MB2 SEGÚN } \\
\text { LATERALIDAD EN MUESTRA, POBLACIÓN ESPECÍFICA DE TEMUCO, CHILE }\end{array}$} \\
\hline \multirow{2}{*}{ Lado } & \multicolumn{2}{|c|}{ MB2 } & \multirow{2}{*}{ Total } & \multirow{2}{*}{ p } \\
\hline & Ausencia & Presencia & & \\
\hline $\begin{array}{l}\text { Femenino } \\
\text { Masculino }\end{array}$ & $\begin{aligned} 11 & (64,7 \%) \\
6 & (35,3 \%)\end{aligned}$ & $\begin{array}{ll}20 & (51,3 \%) \\
19 & (48,7 \%)\end{array}$ & $\begin{array}{ll}31 & (55,4 \%) \\
25 & (44,6 \%)\end{array}$ & 0,353 \\
\hline Total & $17(100,0 \%)$ & $39(100,0 \%)$ & $56(100,0 \%)$ & \\
\hline
\end{tabular}

debido a la incapacidad de localizar el canal MB2 y al desconocimiento de la morfología de éste, lo que impide lograr un correcto desbridamiento u obturación, dando como resultado un mal pronóstico a largo plazo $(8,16)$.

La frecuencia del canal MB2 reportada en la literatura es variable, según la técnica de observación utilizada, así como también si se realizó in vivo o in vitro, con rangos que varían entre el 0 y $100 \%$ (17).

En el presente estudio, se observó una frecuencia del 69,64\% del canal MB2, distribuido en un 51,3\% en mujeres y $48,7 \%$ en hombres, lo que representa una alta frecuencia de esta variación anatómica, coincidiendo con Lee (4), Rathi (5) y Betancourt (18), quienes reportaron la presencia del canal MB2 sobre un $50 \%$. Nuestro porcentaje fue superior al reportado por Weine (19) (51,5\%) y Abuabuara (20) (50\%), quienes utilizaron la misma técnica de localización. No hay reportes de frecuencia del canal MB2 en estudios in vivo a través de radiografías periapicales, debido a la baja sensibilidad en la detección que ofrece esta técnica. Sin embargo, al tomar una ra- diografía con orientación desde distal, con un ángulo horizontal de $15^{\circ}(11)$, permite desproyectar la raíz mesiovestibular de la raíz palatina y así obtener mayor información. Al contrastar nuestros resultados con frecuencias obtenidas a través del examen TCHC, in vitro, son semejantes al $65 \%$ de Campos Netto y cols. (21) 54\% de Abuabuara (19), aunque Bauman (22) reporta un 92\%, lo que demuestra la alta sensibilidad del TCHC como instrumento diagnóstico endodóntico. Hoy en día, el sistema de TCHC permite realizar un análisis adicional de la presencia del canal MB2. Además, reduce significativamente la dosis de radiación, lo cuál la habilita para ser utilizada rutinariamente. Sin embargo, el examen de la morfología dentaria tridimensional preoperatoria de evaluación diagnóstica no se utiliza con frecuencia, y aunque presenta ventajas, la técnica de radiografía periapical es la más utilizada en la práctica clínica.

Weine (6) estudió in vitro la morfología del sistema canalicular de la raíz mesiovestibular en el $1 \mathrm{MM}$, a través de limas endodónticas posicionadas en los canales para luego visualizar los diferentes tipos de configuraciones a través de radiografías periapica- 
Betancourt P, Aracena Rojas S, Navarro Cáceres P, Fuentes R.

les, reportando un $42 \%$ para la clasificación tipo I, porcentaje superior a nuestro $30,38 \%$; observó un $24,2 \%$ para la clasificación tipo II, inferior al $41,07 \%$ señalado en este artículo; tipo III un $30,4 \%$ verus un $28,57 \%$ y $3,4 \%$ tipo IV. Cabe mencionar que el estudio realizado por Weine fue realizado en una muestra mayor $(n=293)$ y en población asiática, lo cuál puede explicar las diferencias con la población sudamericana estudiada en el presente artículo. Algunas diferencias fueron encontradas al contrastar nuestros resultados con los de Weine (18), quién en otro artículo utilizó la técnica de corte transversal, in vitro, observando en el tipo I un $48,5 \%$, versus un $38,38 \%$ reportado en nuestro estudio, en el tipo II un $37,5 \%$ versus un $41,07 \%$ y en el tipo III $14 \%$ versus $28,57 \%$. En ambos estudios, Weine reporta el sistema tipo I como el más prevalente, en contraposición a nuestros resultados, donde es el tipo II.

Resultados similares a los de este estudio son descritos por Gilles (23), quién a través de un estudio in vitro de microscopía electrónica de barrido, reportó que el sistema de canales radiculares tipo II fue el más prevalente con un 52,4\%, y el sistema tipo IV el de menor frecuencia, con un $4,8 \%$.

Fogel (24) publicó en 1994 resultados similares a los expuestos en el presente artículo, utilizando método de radiografías periapicales, in vivo, donde el tipo I mostró un $28,9 \%$, el tipo II un $39,4 \%$, clase III un $31,7 \%$ y clase IV $0 \%$, resultando la forma II la más frecuente.

La clasificación tipo II fue la más frecuente, lo que podría explicar la razón por la cual diversos tratamientos pulpares pueden ser exitosos en primera instancia, aún cuando no se logra localizar el canal MB2, ya que ambos se unen en el tercio apical, para terminar en un canal único, sin embargo, se debe tener en cuenta la variabilidad morfológica canalicular del canal MB2 y su localización para disminuir el porcentaje de los fracasos terapéuticos a mediano o largo plazo, ya que el pronóstico disminuye considerablemente si no se instrumentan ambos canales.

\section{CONCLUSIÓN}

El clínico debe tener la convicción de realizar la exploración en búsqueda del canal MB2, logrando un ade- cuado acceso endodóntico que permita una visualización correcta y utilizar herramientas como el microscopio, ultrasonido o TCHC para su detección y estudio morfológico. Además, se recomienda el uso de la radiografía periapical con deslizamiento, lo cual aumenta la probabilidad de detección clínica del canal MB2.

\section{BIBLIOGRAFÍA}

1. Zheng Q, Wang Y, Zhou X, Wang Q, Zheng G, Huang D. A Cone-Beam Computed Tomography Study of Maxillary First Permanent Molar Root and Canal Morphology in a Chinese Population. J Endod 2010;36:1480-4.

2. Zhang R, Yang H, Yu X, Wang H, Hu T, Dummer PMH. Use of CBCT to identify the morphology of maxillary permanent molar teeth in a Chinese subpopulation. Int Endod J 2011;44:162-9.

3. Baratto F, Zaitter S, Aihara G, Alves E, Abuabara A, María G. Analysis of the internal anatomy of maxillary first molars by using different methods. J Endod 2009;35:337-42.

4. Lee J, Kim K, Lee J, Park W, Jeong J, Lee Y, Gu Y, Chang S, Son W, Lee W, Baek S, Bae K, Kum K. Mesiobuccal root canal anatomy of Korean maxillary first and second molars by cone-beam computed tomography Oral Surg Oral Med Oral Pathol Oral Radiol Endod 2011;111:785-91.

5. Rathi S, Patil J, Jaju PP. Clinical Study Detection of Mesiobuccal Canal in Maxillary Molars and Distolingual Canal in Mandibular Molars by Dental CT: A Retrospective Study of 100 Cases. Int J Dent 2010; 2010:291276.

6. Weine FS, Hayami S, Hata G, Toda T. Canal configuration of the mesiobuccal root of the maxillary first molar of a Japanese sub-population. International Endodontic Journal 1999;32:79-87.

7. Kim Y, Lee SJ, Woo J. Morphology of maxillary first and second molars analyzed by cone-beam computed tomography in a korean population: variations in the number of roots and canals and the incidence of fusion. J Endod 2012;38(8):1063-8. 
8. Wolcott J, Ishley D, Kennedy W, Johnson S, Minnich S. Clinical investigation of second mesiobuccal canals in endodontically treated and retreated maxillary molars. J Endod 2002;28(6):477-9.

9. Yoshioka T, Kikuchi I, Fukumoto Y, Kobayashi C, Suda H. Detection of the second mesiobuccal canal in mesiobuccal roots of maxillary molar teeth ex vivo. Int Endod J 2005;38:124-8.

10. Schwarze T, Baethge C, Stecher T, Geurtsen W. Identification Of Second Canals In The Mesiobuccal Root Of Maxillary First And Second Molars Using Magnifying Loupes Or An Operating Microscope. Aust Endod J 2002;28(2):57-60.

11. Baldassari-Cruz LA, Lilly JP, Rivera EM. The influence of dental operating microscope in locating the mesiolingual canal orifice. Oral Surg Oral Med Oral Pathol Oral Radiol Endod 2002;93 (2):190-4.

12. Alaçam T, Cemal AC, Genç Ö, Kayaoglu G. Second mesiobuccal canal detection in maxillary first molars using microscopy and ultrasonics. Aust Endod J 2008;34:106-9.

13. Somma, F, Leoni D, Plotino D, Grande NM, Plasschaert A. Root canal morphology of the mesiobuccal root of maxillary first molars: a microcomputed tomographic analysis. Int Endod J 2008;42:165-74.

14. Gorduysus O M, Gorduysus M, Friedman S. Operating Microscope Improves Negotiation of Second Mesiobuccal Canals in Maxillary Molars. J Endod 2001;27(11):683-6.

15. Betancourt P, Aracena D, Bustos D. Estudio comparativo in vitro de la calidad de obturación del sistema ProTaper Universal Manual, versus el sistema de condensación lateral. Int. J. Odontostomat 2011;5(1):49-53.

16. Vertucci J. Root canal anatomy of the human permanent teeth. ORALSURC 1984;58:589-99.

17. Cleghorn B, Christie W, Dong C. Root and root canal morphology of the human permanent maxillary first molar: a literature review. J Endod 2006;32:813-21.
18. Betancourt P, Fuentes R, Aracena Rojas S, Cantín M, Navarro Cáceres P. Prevalencia del segundo canal en la raíz mesiovestibular de los primeros molares maxilares mediante tomografía computarizada de haz de cono. Av. Odontoestomatol 2013;29(1):31-6.

19. Weine FS, Healey HJ, Gerstein H, Evanson L. Canal configuration in the mesiobuccal root of the maxillary first molar and its endodontic significance. J Endod 2012;38(10):1305-8.

20. Abuabara A, Baratto-Filho F, Aguiar Anele J, Leonardi DP, Sousa-Neto MD. Efficacy of clinical and radiological methods to identify second mesiobuccal canals in maxillary first molars. Acta Odontol Scand 2013;71(1):205-9.

21. Campos Netto P A, Lins C C S A, Lins C V, Lima G A, Frazao M A G. Study of the internal morphology of the mesiobuccal root of upper first permanent molar using cone beam computed tomography. Int J Morphol 2011;29 (2):617-21.

22. Bauman R, Scarfe W, Clark S, Morelli J, Scheetz J, Farman A. Ex vivo detection of mesiobuccal canals in maxillary molars using CBCT at four different isotropic voxel dimensions. Int Endod $\mathrm{J}$ 2011;44(8):752-8.

23. Gilles J, Reader A. An SEM investigation of the mesiolingual canal in human maxillary first and second molars. Al surc oral med oral pathol 1990; 70:638-43.

24. Fogel HM, Peikoff MD, Christie. Canal configuration in the mesiobuccal root of the maxillary first molar: A clinical study. J Endod 1994;20(3):135-7.

\section{CORRESPONDENCIA}

Pablo Betancourt

Departamento Integral Adultos

Facultad de Odontología

Universidad de La Frontera

Manuel Montt, 112, Temuco, Chile.

E-mail: pablo.betancourt@ufrontera.cl 